\title{
flimview : A software framework to handle, visualize and
}

\section{analyze FLIM data [version 1; peer review: 1 approved, 1}

\section{approved with reservations]}

\author{
Matias Carrasco Kind ${ }^{1}$, Mantas Zurauskas 2,3, Aneesh Alex², \\ Marina Marjanovic 2,3,5,6, Prabuddha Mukherjee1,2, Minh Doan4, \\ Darold R. Spillman Jr. 2,3, Steve Hood2,7, Stephen A. Boppart (iD),3,5,6,8
}

\footnotetext{
${ }^{1}$ National Center for Supercomputing Applications, University of Illinois at Urbana-Champaign, Urbana, IL, 61801, USA

2GSK Center for Optical Molecular Imaging, University of Illinois at Urbana-Champaign, Urbana, IL, 61801, USA

${ }^{3}$ Beckman Institute for Advanced Science and Technology, University of Illinois at Urbana-Champaign, Urbana, IL, 61801, USA

${ }^{4}$ GlaxoSmithKline, Collegeville, PA, USA

${ }^{5}$ Department of Bioengineering, University of Illinois at Urbana-Champaign, Urbana, IL, 61801, USA

${ }^{6}$ Carle Illinois College of Medicine, University of Illinois at Urbana-Champaign, Urbana, IL, 61801, USA

${ }^{7}$ GlaxoSmithKline, Stevenage, UK

${ }^{8}$ Department of Electrical and Computer Engineering, University of Illinois at Urbana-Champaign, Urbana, IL, 61801, USA
}

V1 First published: 08 Jun 2020, 9:574

https://doi.org/10.12688/f1000research.24006.1

Latest published: 08 Jun 2020, 9:574

https://doi.org/10.12688/f1000research.24006.1

\section{Abstract}

flimview is a bio-imaging Python software package to read, explore, manage and visualize Fluorescence-Lifetime Imaging Microscopy (FLIM) images. It can open the standard FLIM data file conventions (e.g., sdt and ptu) and processes them from the raw format to a more readable and manageable binned and fitted format. It allows customized kernels for binning the data as well as user defined masking operations for pre-processing the images. It also allows customized fluorescence decay fitting functions and preserves all of the metadata generated for provenance and reproducibility.

Outcomes from the analysis are lossless compressed and stored in an efficient way providing the necessary open-source tools to access and explore the data. flimview is open source and includes example data, example Jupyter notebooks and tutorial documentation. The package, test data and documentation are available on Github.

\section{Keywords}

FLIM, bio-imaging, microscopy, visualization, fluorescence, Python

This article is included in the Python collection.

\section{Open Peer Review \\ Approval Status $\checkmark$ \\ 1 2 \\ version 1

$\checkmark$
view \\ 1. Xavier Michalet (D), University of California, Los Angeles (UCLA), Los Angeles, USA \\ 2. Nirmal Mazumder (D), Manipal School of Life Sciences, Manipal Academy of Higher Education (MAHE), Manipal, India} Any reports and responses or comments on the article can be found at the end of the article. 
Corresponding author: Matias Carrasco Kind (mcarras2@illinois.edu)

Author roles: Carrasco Kind M: Conceptualization, Software, Visualization, Writing - Review \& Editing; Zurauskas M: Conceptualization, Methodology, Validation, Writing - Review \& Editing; Alex A: Conceptualization, Project Administration, Writing - Review \& Editing;

Marjanovic M: Conceptualization, Writing - Review \& Editing; Mukherjee P: Conceptualization, Methodology, Writing - Review \& Editing; Doan M: Conceptualization, Supervision; Spillman Jr. DR: Resources, Supervision; Hood S: Supervision; Boppart SA: Supervision

Competing interests: S.A.B receives research grant support from GlaxoSmithKline related to the research described here, and reports receiving consulting fees from and owning equity interest in PhotoniCare, Inc., Diagnostic Photonics, Inc. and LiveBx, LLC. A.A., M.D. and S.H. are employees and shareholders of GlaxoSmithKline. M.Z, M.M., P.M., D.R.S. declare no potential conflict of interest.

Grant information: The work presented here was funded by GlaxoSmithKline.

The funders had no role in study design, data collection and analysis, decision to publish, or preparation of the manuscript.

Copyright: ( 2020 Carrasco Kind $\mathrm{M}$ et al. This is an open access article distributed under the terms of the Creative Commons Attribution License, which permits unrestricted use, distribution, and reproduction in any medium, provided the original work is properly cited.

How to cite this article: Carrasco Kind M, Zurauskas $M$, Alex A et al. flimview : A software framework to handle, visualize and analyze FLIM data [version 1; peer review: 1 approved, 1 approved with reservations] F1000Research 2020, 9:574 https://doi.org/10.12688/f1000research.24006.1

First published: 08 Jun 2020, 9:574 https://doi.org/10.12688/f1000research.24006.1 


\section{Introduction}

Fluorescence lifetime imaging microscopy (FLIM) is an imaging technique where the image contrast is derived from the differences in the exponential decay rate of the fluorescence from a fluorescent sample (Bower et al., 2018).

In modern two-photon FLIM, the most widely used detection method is known as time-correlated single-photon counting (TCSPC) (Becker et al., 2004). TCPC employs a pulsed excitation source, such as a laser or a light emitting diode and measures the timing of arrival of single photons originating from the fluorescent sample on a detector to reconstruct the fluorescence lifetime decay (McGinty et al., 2016). Typically, different fluorophores can be characterized by their fluorescence lifetimes. Furthermore, fluorescence lifetimes of a fluorophore can be affected by its environment, therefore FLIM have emerged as a valuable tool, providing unique contrast mechanisms for biomedical imaging (Bower et al., 2019; Hirvonen \& Suhling, 2016; Ranawat et al., 2019).

To reconstruct TCSPC based FLIM image, several non-trivial data processing steps are necessary. For extracting the lifetime and amplitude data from initially saved raw files, first the data has to be imported from proprietary files such as Becker and Hickl . sdt, or PicoQuant .ptu. Next, signal preprocessing through denoising or binning has to be applied to ensure robust performance in low photon count scenarios. Finally, after compensating for instrument response function, biexponential decay curves are fitted to extract lifetime and amplitude values for two dominant components at each pixel.

Several commercial and open source packages are already available for FLIM analysis (Bergmann, 2003; Fli; Warren et al., 2013). However, in practice, when custom FLIM analysis capabilities are required, this can lead to a need for building complicated signal processing and analysis pipelines (Borhani et al., 2019; Bower et al., 2017; Cao et al., 2020), involving a mix of commercial tools, open-source software and bespoke analysis algorithms. While other packages have been published, (Ballesteros et al., 2019), we expect that flimview, with its focus on simplicity, and versatility, will serve as a useful tool for the FLIM community which can also contribute to its growth.

Here we present a Python package, flimview, which provides a user-friendly toolkit for opening, fitting and visualizing FLIM data. The package can be used as a stand-alone tool for analyzing and visualizing FLIM data, or it can be used as a basis for creating end-to-end streamlined analytical pipelines in Python.

\section{Methods}

Implementation

flimview is a library module implemented in Python 3 and can be installed using PyPI (e.g., pip install flimview). Installation instructions using Conda are also included within the repository. A complete list of requirements can be found at the package Github page but include standard Python libraries such as pandas, scipy, matplotlib, h5py, etc. The code is released under the open-source NCSA license which is based on the MIT open source license, allowing full and free use for both commercial and non-commercial purposes. Full usage documentation, Jupyter notebooks (web-browser interface that allows live Python codes), test data and others, including guidelines for contributions and issue reporting, are included in the project repository. This package has been tried in Windows, Mac OS and Linux Operating Systems. In general flimview works better in the latter two for which it is also expected to get supported development.

\section{Operation}

f limview consists of several utility libraries for storage, visualization and manipulation of FLIM data. After reading and processing the raw data (.ptu or . sdt files), the input data is stored in the main class of the package, a Flimcube, which is an object that represents $3 \mathrm{D}$ data (2D spatial dimensions and one temporal dimension) allowing multiple methods to access and manipulate the data cube.

Among its attributes is the header which includes all the metadata available for the dataset, sizes of the arrays, resolution and whether this cube is binned and/or masked (see Binning and Masking sections below). When the data cube is masked, a process described in the following sections, the pixel-level mask is also included as an attribute in the FlimCube object. Due to its construction, we consider a FlimCube to be self-explanatory as it contains all needed information (including its corresponding metadata) to analyze, visualize and fit the data.

Binning. flimview includes a binning function with several pre-defined kernels, although a user-defined kernel is also possible and can be easily added. The binning procedure is simply a convolution kernel applied to the 
image to increase the signal or to enhance its features. This function takes a FlimCube as an input and returns a FlimCube as output by copying all of the metadata and properties from the input data. The following snippet shows an example on how to bin an existing FlimCube using a Gaussian kernel of size $9 \times 9$ and a sigma of 3 (the size of the kernel is $2 * \mathrm{~b}+1$, where $b$ is the bin size)

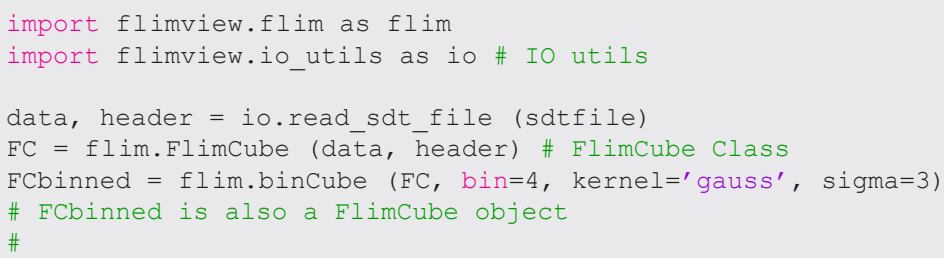

The kernel functions included in $\mathrm{flimview}$ are Gaussian kernel, Airy disk kernel, linear kernel, and a flat kernel. Customized kernels can be easily incorporated. Figure 1 shows examples of different $9 \times 9$ kernels where we can observe how the weight is distributed around the central pixel. In the case of a Gaussian kernel or an Airy disk kernel, the width of the kernel can be customized using the sigma $\sigma$ parameter. For reference, the Gaussian kernel is given by:

$$
I(x)=I_{0} e^{-x^{2} / 2 \sigma^{2}}
$$

where $x$ is the distance to the center pixel and $I_{0}$ is a normalization factor (usually just a unit). In the case of the Airy disk, the kernel is given by:

$$
I(x)=I_{0}\left[\frac{2 J_{1}(x)}{x}\right]^{2}
$$

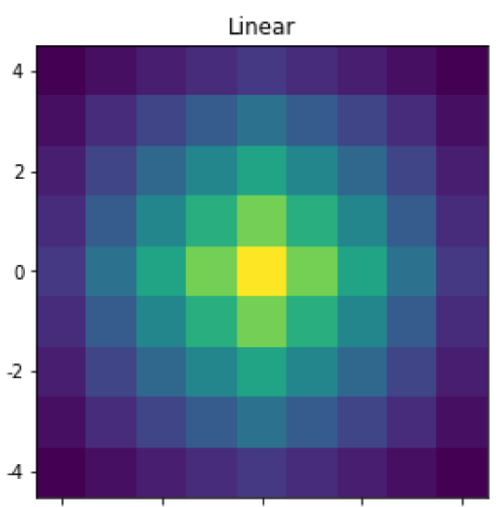

Gaussian, $\sigma=2.5$

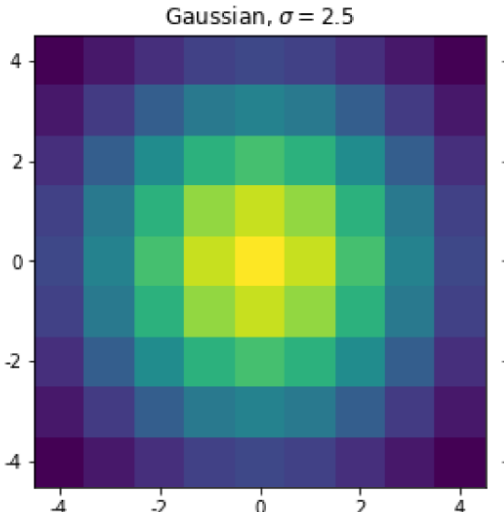

Gaussian, $\sigma=5 / 3$

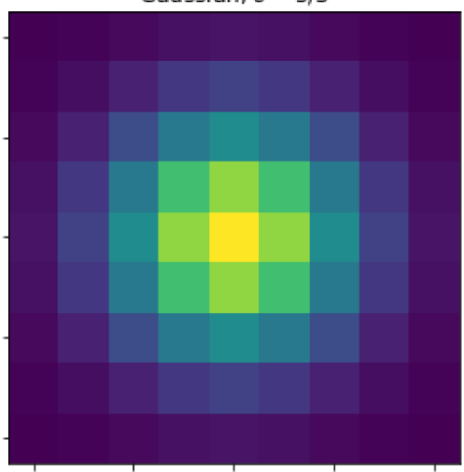

Airy Disk, $\sigma=5 / 3$

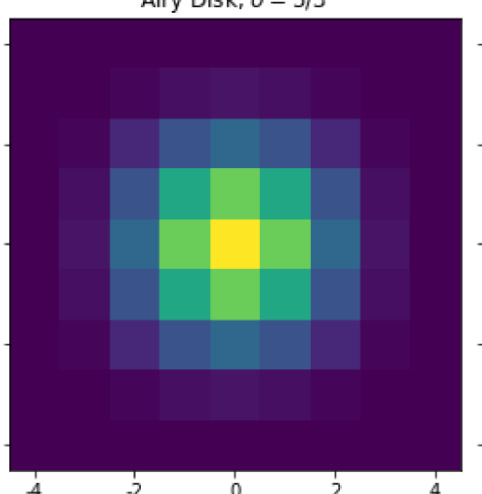

Gaussian, $\sigma=8$

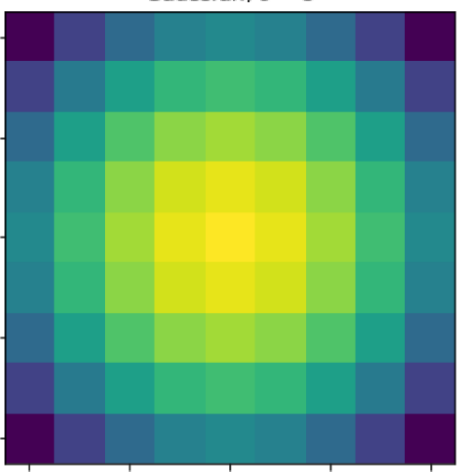

Airy Disk, $\sigma=8$

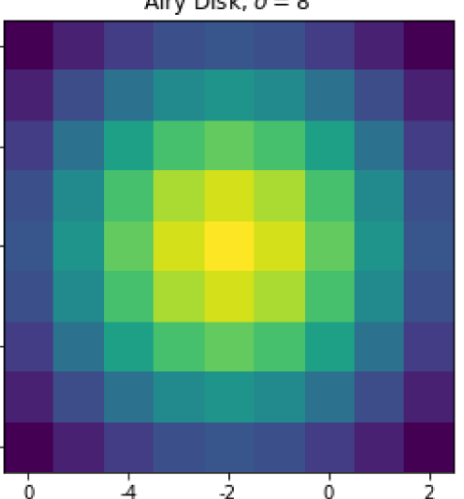

Figure 1. Example of different $9 \times 9$ kernels used for binning. 
where $J_{1}(x)$ is the Bessel function of the first kind of order one. In both cases, $I(x)$ is discretized and normalized to the unit across the $2 \mathrm{~d}$ binning window.

Masking. FlimCube also includes methods for looking at the header information and for masking pixels below a given integrated intensity, or below a given peak threshold in the time series, or even for a custom made geometry mask. The pixels masked this way will not be used during the fitting analysis while the mask is also saved within the same input FlimCube object. The following snippet shows how to apply a mask for a given FlimCube :

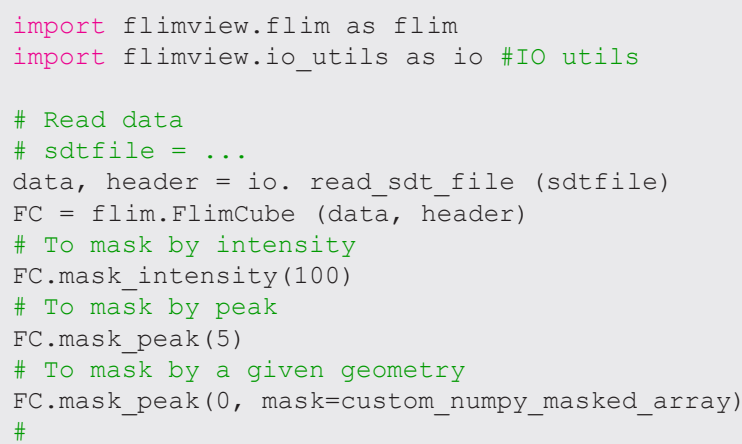

Figure 2 shows different masks applied to the example raw (top) and binned (bottom) data. Masks can be combined and can be transferred between binned and raw data.

Fitting. Once the data is read and binned, and have the low signal and error pixels masked, flimview provides a function to fit a decay function for every pixel in the temporal data using a predefined model (from the module models. py which can be customized and also parallelized. By default a double exponential is used as follows:

$$
f(t)=a_{1} e^{-t / \tau_{1}}+a_{2} e^{-t / \tau_{2}}+l_{0}
$$

where $a_{1}$ and $a_{2}$ are the amplitudes for the given exponential factor. Usually, there is an extra constraint given by $a_{1}+a_{2}=1$. $\tau_{1}$ and $\tau_{2}$ are the mean lifetime for each exponential. $l_{0}$ is a level constant.

Before fitting the entire cube, a single fit is done to the mean intensity values to obtain initial guess parameters for the fitting procedures as shown in Figure 3, as well as the boundaries to each parameter which can also be provided by hand. The snippet below shows how this is done using $f l$ imview :
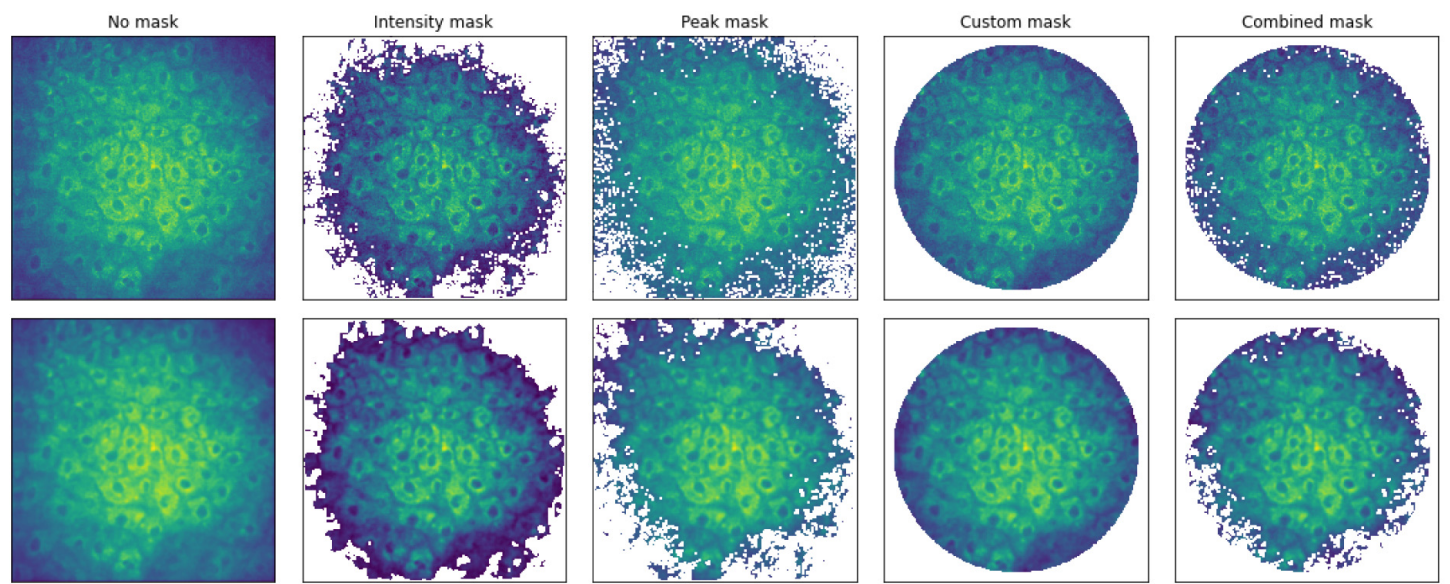

Figure 2. Different Masking approaches to the raw data (top) and binned data (bottom). Masks can be combined. Masked pixels will not be used in the analysis. 


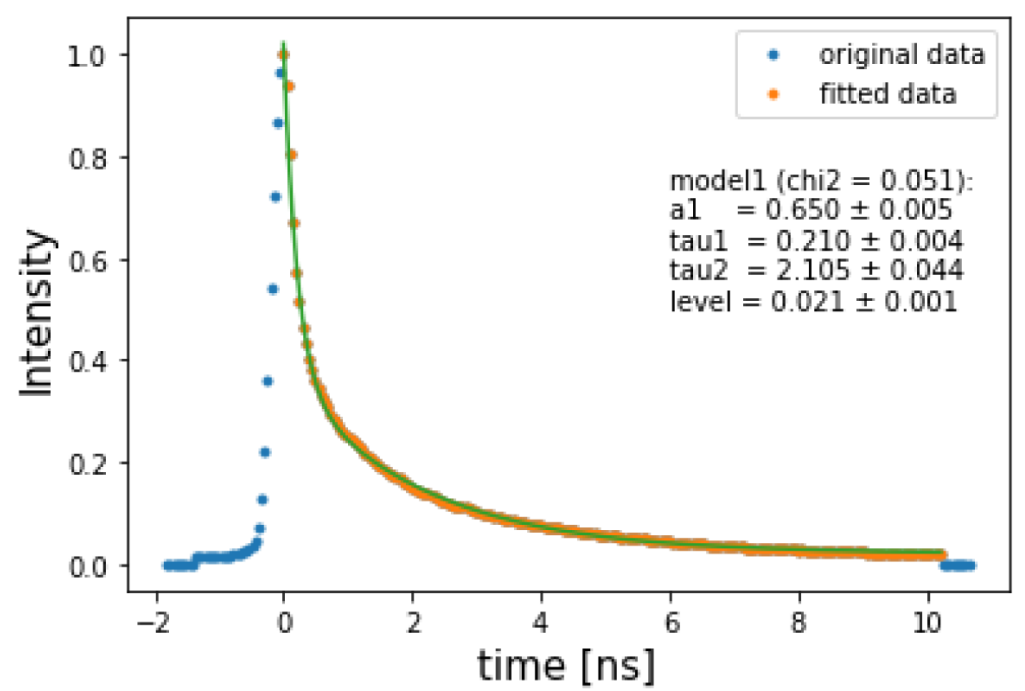

Figure 3. Example of fitting the average and normalized pixel decay to determine initial parameters.

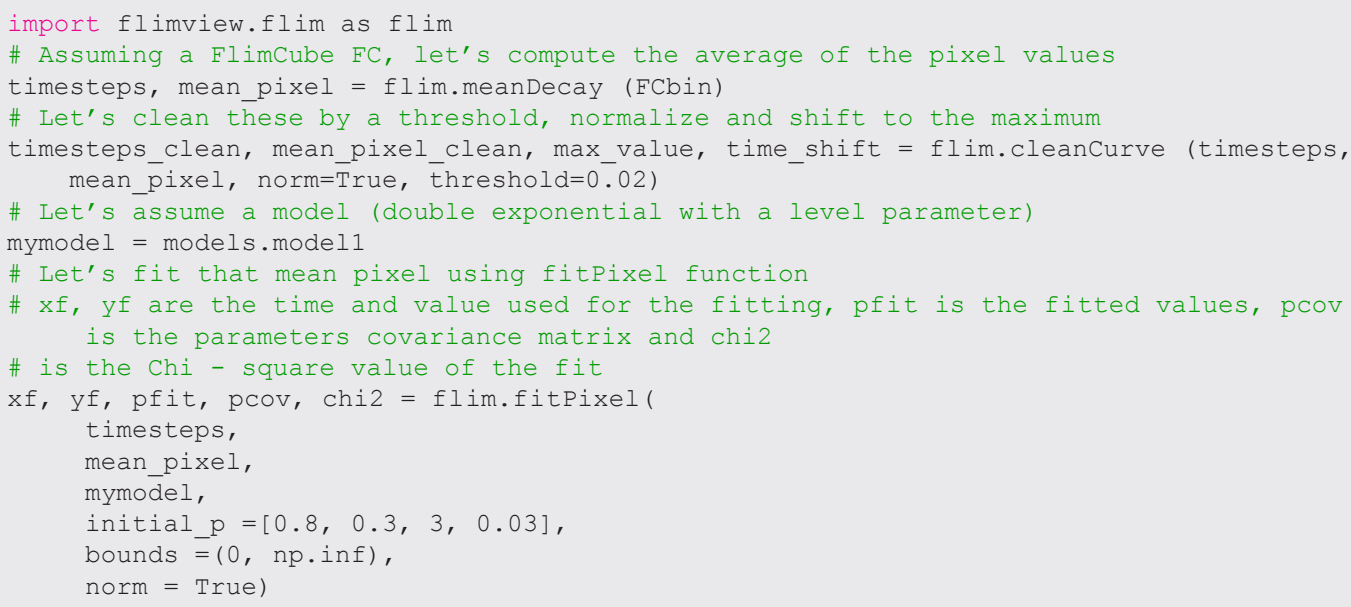

Note that the data is normalized, cleaned, and shifted with respect its peak. For example, to generate Figure 3 we can use the following:

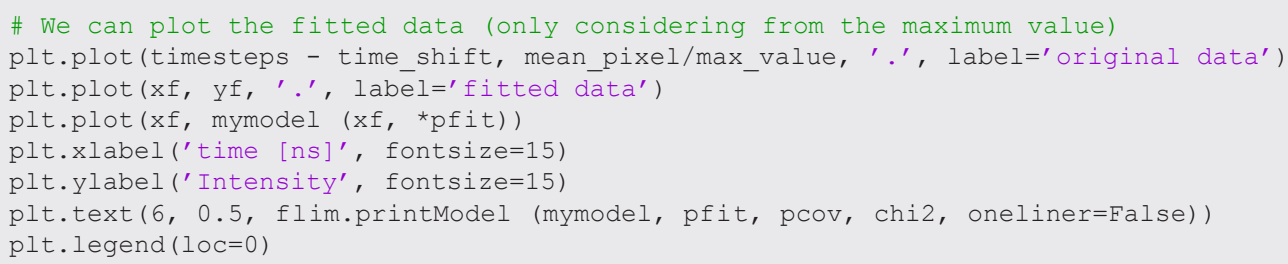

After those parameters are defined and a model is selected a function fitCube takes a FlimCube as input and produces another flimview class called FlimFit which contains the fitting information for every pixel, 
including all parameters from the model, their errors and $\chi^{2}$ values. It is a collection of $2 \mathrm{D}$ arrays for the results of the fitting.

To generate a FlimFit object we can use the following:

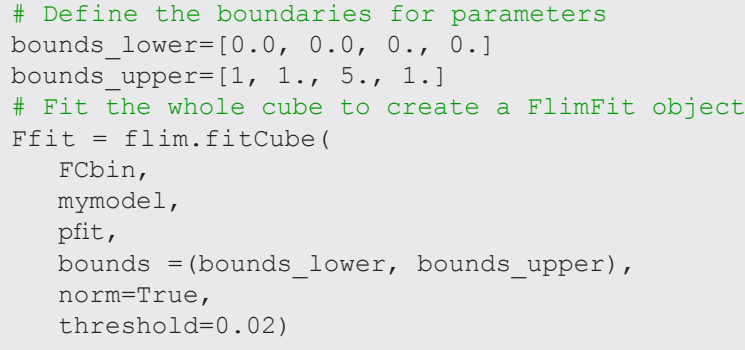

Visualization. The package also includes means to visualize the FlimCube and FlimFit objects using intensity or peak values. Additionally, one can visualize the results from the fitting procedure using a function called plotFit, which takes a FlimCube and a FlimFit as input, along with a pixel coordinate, to produce a figure as the one shown in Figure 4, showing the fit results for a given pixel, the parameters, the residuals, and the residuals distribution which is a useful way to explore the results. These can be combined in an interactive widget showing, for example, the same figure for a given point selected interactively. It is also possible to combine multiple visualizations to generate a sequence of plots into an animation of the datacube. The package repository includes examples on how this can be done to generate a short animation. Other visualization functions are also included and documented in the package.

Storage. FlimCube and FlimFit can be easily stored and easily retrieved by using a hierarchical and compression data store format for array-like data called HDF5 (The HDF Group, 2000-2020). Using this data format, we can store multiple data in different 'folders', including parameters, different models, masks, raw, binned and fitted data in one file for easy retrieval, as it only loads what is asked for, saving memory consumption if necessary. In the case of multiple files, we can serve them on-demand using a web server and only load the data as necessary, including over the web, which is an HDF5 feature. The methods saveCube, saveFit, loadCube, loadFit, viewH5 are implemented in the io_utils modules inside flimview. For example, after running f i tCube and saving the example data in a file, one can easily see its internal structure with:

import flimview.io utils as io

io.viewH5 (h5file)

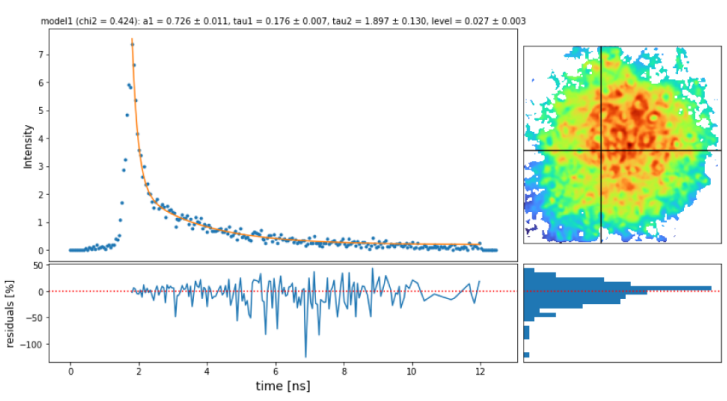

(a) SDT file

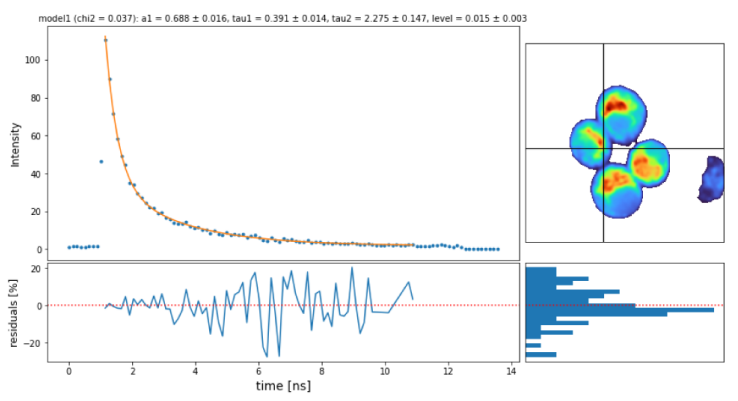

(b) PTU file

Figure 4. Visualization example showing the fit for an individual pixel, the residuals and the location on the image for both, . sdt (a) and .ptu (b) files. 
which produces the following output, showing the file content of the processed data:

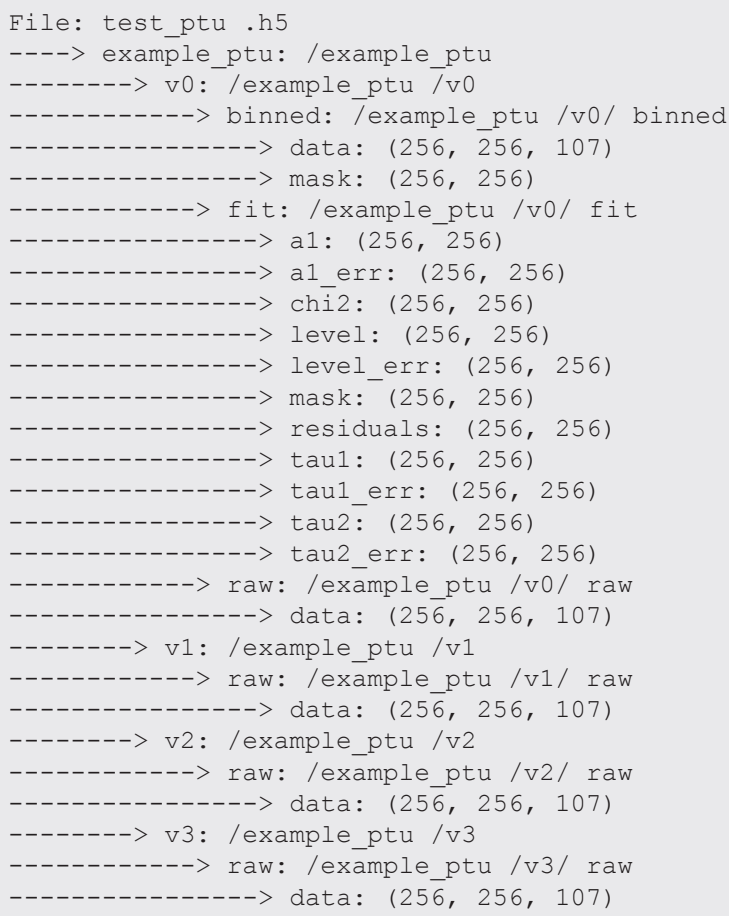

More examples on how to save and retrieve FLIM data using this format are included in the example notebooks at the Github repository.

\section{Use cases}

To highlight the main features of flimview, we have used two of the most common FLIM data formats, namely, the Becker and Hickl . sdt, and PicoQuant . ptu. We have included the reading routines for both data types but others can be easily extended within the module. All the figures in this paper were made using the two example files included in the package which we describe below. In particular Figure 4 shows both files presented in the same visualization after being processed into a Flimcube. Both example files are included with the package and are briefly described below.

\section{SDT File}

The example file epidermis.sdt contains 2-photon excited FLIM data from the skin (epidermis) on the upper forearm of a healthy human volunteer captured with commercial optical medical imaging system (MPT-lex CARS, JenLab GmbH, Germany) (Weinigel et al., 2015). The image was captured using the procedure described in (Alex et al., 2018). For generating optical images, the excitation wavelength of the femtosecond laser was set to $760 \mathrm{~nm}$ and the incident in situ laser power was set to $30 \mathrm{~mW}$. The light was focused through a 40x 1.35 NA objective. Autofluorescence signals within the spectral range of $405 \mathrm{~nm}-600 \mathrm{~nm}$ were detected. All these parameters are extracted from the file and added to the header inside the FlimCube.

\section{PTU File}

The example file macrophages.ptu contains the 2-photon excited FLIM data from J774A.1 mouse macrophages grown in Dulbecco's Modified Eagle Medium $+10 \%$ FBA $+1 \%$ antibiotic, under $5 \% \mathrm{CO}_{2}$. The image was acquired with the laser set to $750 \mathrm{~nm}$ and the incident in situ laser power was set to $25 \mathrm{~mW}$.

\section{Summary}

In this paper we have presented flimview, a novel Python 3 package to manipulate, visualize, and analyze FLIM images and measurements from specific data formats . sdt and . ptu. We included snippets on how different 
kernels and different masking strategies can be applied after reading the raw data into a FlimCube object. We also show how the procedure of fitting the whole cube is carried out and how these results can be visualized effectively. Most of the functions provided in this package can be customized for further research or to fit specific needs, including user defined binning kernels, mask functions or exponential decay fitting models. We showed how, using a hierarchical data format, the results from multiple files, multiple formats and multiple views can be stored in a single optimized data format called HDF5 which allows much better data management and data access to the data sets, even in the case of multiple files.

\section{Data availability}

We have included two data examples along with Jupyter notebooks to explore and get started with the module and its functions. This data examples can be found in the following repository: https://github.com/BiophotonicsCOMI/flimview. Individual files can be loaded locally using this snippet. Detailed instructions and description of the data included can be found in the package website:

from flimview import datasets

\# Get SDT example file

datasets.fetch_sdt()

\# Get PTU file

datatsets.fetch_ptu()

\section{Software availability}

1. Package and installation instructions can be found here: https://github.com/Biophotonics-COMI/flimview

2. Source code can be found here: https://github.com/Biophotonics-COMI/flimview

3. Archived source code at time of publication: http://doi.org/10.5281/zenodo.3825694 (Carrasco Kind, 2020).

4. License: NCSA

Alex A, Frey S, Angelene $\mathrm{H}$, et al.: In situ biodistribution and residency of a topical anti-inflammatory using fluorescence lifetime imaging microscopy. Br J Dermatol. 2018; 179(6): 1342-1350.

PubMed Abstract | Publisher Full Text

Ballesteros GC, Proux R, Bonato C, et al:: readPTU: a python library to analyse time tagged time resolved data. Journal of Instrumentation. 2019; 14(06): T06011-T06011.

Publisher Full Text

Becker W, Bergmann A, Hink MA, et al.: Fluorescence lifetime imaging by time-correlated single-photon counting. Microsc Res Tech. 2004; 63(1): 58-66.

PubMed Abstract | Publisher Full Text

Bergmann A: Spcimage: data analysis software for fluorescence lifetime imaging microscopy. Becker \& Hickl GmbH. 2003.

Reference Source

Borhani N, Bower AJ, Boppart SA, et al.: Digital staining through the application of deep neural networks to multi-modal multiphoton microscopy. Biomed Opt Express. 2019; 10(3): 1339-1350. PubMed Abstract | Publisher Full Text | Free Full Text

Bower AJ, Li J, Chaney EJ, et al.: High-speed imaging of transient metabolic dynamics using two-photon fluorescence lifetime imaging microscopy. Optica. 2018; 5(10): 1290-1296. PubMed Abstract | Publisher Full Text | Free Full Text

Bower AJ, Marjanovic M, Zhao Y, et al.: Label-free in vivo cellular-level detection and imaging of apoptosis. J Biophotonics. 2017; 10(1): 143-150.

PubMed Abstract | Publisher Full Text | Free Full Text

Bower AJ, Sorrells JE, Li J, et al:: Tracking metabolic dynamics of apoptosis with high-speed two-photon fluorescence lifetime imaging microscopy. Biomed Opt Express. 2019; 10(12): 6408-6421. PubMed Abstract | Publisher Full Text | Free Full Text
Carrasco Kind M: Biophotonics-COMI/flimview: flimview 1.2.0 (Version v1.2.0). Zenodo. 2020

http://www.doi.org/10.5281/zenodo.3825694

Cao R, Wallrabe $\mathrm{H}$, Siller $\mathrm{K}$, et al:: Optimization of FLIM imaging, fitting and analysis for auto-fluorescent $N A D(p) h$ and FAD in cells and tissues. Methods Appl Fluoresc. 2020; 8(2): 024001.

PubMed Abstract | Publisher Full Text

Flimlib: a package for exponential curve fitting of fluorescence lifetime image data. Accessed: 2020-02-26.

Reference Source

Hirvonen LM, Suhling K: Wide-field TCSPC: methods and applications. Meas Sci Technol. 2016; 28(1): 012003. Publisher Full Text

McGinty J, Talbot CB, Owen D, et al.: Fluorescence lifetime imaging: Microscopy, endoscopy, and tomography. In: Handbook of Biomedical Optics. CRC Press, 2016; 609-636. Reference Source

Ranawat $\mathrm{H}, \mathrm{Pal} \mathrm{S}$, Mazumder N: Recent trends in two-photon auto-fluorescence lifetime imaging ( $2 \mathrm{p}$ - flim) and its biomedical applications. Biomed Eng Lett. 2019; 1-18.

PubMed Abstract | Publisher Full Text | Free Full Text The HDF Group: Hierarchical data format version 5, 2000-2020. Reference Source

Warren SC, Margineanu A, Alibhai D, et al.: Rapid global fitting of large fluorescence lifetime imaging microscopy datasets. PLOS One. 2013; 8(8): e70687.

PubMed Abstract | Publisher Full Text | Free Full Text

Weinigel M, Georg Breunig H, Uchugonova A, et al:: Multipurpose nonlinear optical imaging system for in vivo and ex vivo multimodal histology. J Med Imaging. 2015; 2(1): 016003. PubMed Abstract | Publisher Full Text | Free Full Text 


\section{Open Peer Review}

\section{Current Peer Review Status:}

\section{Version 1}

Reviewer Report 01 September 2020

https://doi.org/10.5256/f1000research.26482.r68967

(C) 2020 Mazumder N. This is an open access peer review report distributed under the terms of the Creative Commons Attribution License, which permits unrestricted use, distribution, and reproduction in any medium, provided the original work is properly cited.

\section{Nirmal Mazumder}

Department of Biophysics, Manipal School of Life Sciences, Manipal Academy of Higher Education (MAHE), Manipal, Karnataka, India

The manuscript on flimview is well written and rationale of developing a new software is needed to analysis FLIM data acquired by commercial software or hardware.

The manuscript can be accepted for indexing after minor revision.

Line 4, in introduction, It should be "TCSPC".

In this manuscript, definition of "fluorescence lifetime" is missing.

Author could describe in brief about the instrumentation. Is flimview applicable for single photon or two photon fluorescence measurement?

A step by step flowchart about the flimview could help to implement.

In figure 2, only center of the image is used for analysis after masking. However, in some cases cells are distributed in full field of view. In such case, how does this kind of masking work?

Author should mention in figure 2, what kind of image it is, what does the color mean, what type of cell is imaged.

In fitting, why two lifetimes are used for fitting? If there is only one flurophore then single exponential fitting may work. In case of $\mathrm{NADH}$, it exists in free and bound forms and hence two lifetimes. Author should comment on it.

In figure 4, it will be more acceptable if author can compare the results of flimview with Becker and Hickl or PicoQuant software analysis. Residues should be close to 1. Is it due to experimental or software error?

\section{Is the rationale for developing the new software tool clearly explained?}

Yes 
Is the description of the software tool technically sound?

Partly

Are sufficient details of the code, methods and analysis (if applicable) provided to allow replication of the software development and its use by others?

Yes

Is sufficient information provided to allow interpretation of the expected output datasets and any results generated using the tool?

Partly

Are the conclusions about the tool and its performance adequately supported by the findings presented in the article?

Partly

Competing Interests: No competing interests were disclosed.

Reviewer Expertise: Biophotonics, Biophysics

I confirm that I have read this submission and believe that I have an appropriate level of expertise to confirm that it is of an acceptable scientific standard, however I have significant reservations, as outlined above.

Reviewer Report 02 July 2020

https://doi.org/10.5256/f1000research.26482.r65063

(c) 2020 Michalet X. This is an open access peer review report distributed under the terms of the Creative Commons Attribution License, which permits unrestricted use, distribution, and reproduction in any medium, provided the original work is properly cited.

\section{Xavier Michalet}

Department of Chemistry and Biochemistry, University of California, Los Angeles (UCLA), Los Angeles, CA, USA

Reviewer's Report on the manuscript "flimview..." by Kind et al.

This paper describes a python package to load and visualize FLIM datasets. The source code has been deposited on Github, together with two example data files used to illustrate some of the software features.

The manuscript is well written and describes the software features with a fair amount of detail. The samples of code I have reviewed appear to be also reasonably well documented. Based on these basic criteria, this work should be valuable as a basis for future developments by researchers interested in developing their own tools for FLIM analysis.

I would however encourage the authors to address the following remarks:

\section{Presentation:}


For whatever reasons, the figures do not include color scales, which are present in the Jupyter notebooks used to generate them. This can easily be fixed and would be useful.

The datasets shown correspond to microscopy images of biological samples. It is customary (and very useful) to show such data with a scale bar. Consider making the extra effort to generate one (maybe optionally) as part of the code, to encourage scientific rigor.

Some of the features of the software are incompletely described. For instance, the package opens .sdt and .ptu files containing photon timestamps, but does not dwell on the choice of timestamp binning used internally. This of course has an effect on RAM resources but also potentially on decay artifacts. As a side comment, the package, as is, does not support pre-binned datasets, as far as I can tell (see below). Some .sdt files are pre-binned only and would not be handled by the current software version.

I am not sure what the authors mean by "after compensating for the instrument response function" ( $p 3$ )? Clearly the code does fit the decays without IRF convolution (which of course requires the user to provide an IRF file as well).

While I understand why binning the original dataset could be interesting to increase SNR and decrease data footprint, I am unclear about the role of convolution with Gaussian or other kernels. What would this correspond to physically? I would understand deconvolution of the image with such kernels (although that would not address the question of how the photon time stamps should be redistributed in the deconvolved image), but the purpose of these kernels, besides image beautification, is mysterious and not an encouraged practice for rigorous analysis.

\section{Content:}

While this software is understandably only offering basic features (opening datasets 2 kinds only supported, pre-processing, displaying an intensity image and fitting), it seems to be missing what I would consider an expected functionality for a software bearing the flimview name, namely visualization of fluorescence lifetime information. A barebone commercial software such as SPCImage from Becker \& Hickl, at least does offer a mean lifetime representation, which would not seem to be difficult to implement. Since we are dealing with a software intended for research, I would have naively expected that options to represent the bi-exponential fitted amplitudes and lifetimes as color-coded maps would have been offered.

The software allows saving the data in the open and multiplatform HDF5 format. While this is commendable, it is not quite clear what principles are governing the structure of the data in this file format, or for that matter, what the file format actually looks like from a bird's eye view. Our group has made an attempt to offer a similar type of HDF5-based file format for a slightly different purpose (photon-HDF5), which could be taken as an example (if maybe extreme in its level of detail) of what could be provided to potential users ${ }^{1}$. More importantly, it is not quite clear how this file format will and could evolve, whether it is intended to become a standard, while it has been chosen preferentially to another, etc. A minimum discussion of these 
considerations within the manuscript, and a more extended presentation online (say on the Github website) would be extremely useful.

It would be useful to hear from the authors whether and how they intend to encourage contribution and ensure evolution of the software. Of particular importance would be evidence that there is some kind of quality control (test modules - e.g. such as those described in a software cited in the file loading code, FRETBurst ${ }^{2}$ - written using pytest).

Also related to the lack of bird's eye view description is the absence of discussion of the principles involved in defining the FlimCube class. How can it be extended to support more than one spectral channel (or any other spectroscopic variable)? An additional spatial dimension? An additional temporal dimension?

\section{References}

1. Ingargiola A, Laurence T, Boutelle R, Weiss S, et al.: Photon-HDF5: An Open File Format for Timestamp-Based Single-Molecule Fluorescence Experiments.Biophys J. 2016; 110 (1): 26-33 PubMed Abstract | Publisher Full Text

2. Ingargiola A, Lerner E, Chung S, Weiss S, et al.: FRETBursts: An Open Source Toolkit for Analysis of Freely-Diffusing Single-Molecule FRET.PLoS One. 2016; 11 (8): e0160716 PubMed Abstract | Publisher Full Text

Is the rationale for developing the new software tool clearly explained? Yes

Is the description of the software tool technically sound? Yes

Are sufficient details of the code, methods and analysis (if applicable) provided to allow replication of the software development and its use by others?

Yes

Is sufficient information provided to allow interpretation of the expected output datasets and any results generated using the tool?

Yes

Are the conclusions about the tool and its performance adequately supported by the findings presented in the article?

Yes

Competing Interests: No competing interests were disclosed.

Reviewer Expertise: single-molecule FRET, wide-field FLIM, single-photon detectors

I confirm that I have read this submission and believe that I have an appropriate level of expertise to confirm that it is of an acceptable scientific standard. 
The benefits of publishing with F1000Research:

- Your article is published within days, with no editorial bias

- You can publish traditional articles, null/negative results, case reports, data notes and more

- The peer review process is transparent and collaborative

- Your article is indexed in PubMed after passing peer review

- Dedicated customer support at every stage

For pre-submission enquiries, contact research@f1000.com 